
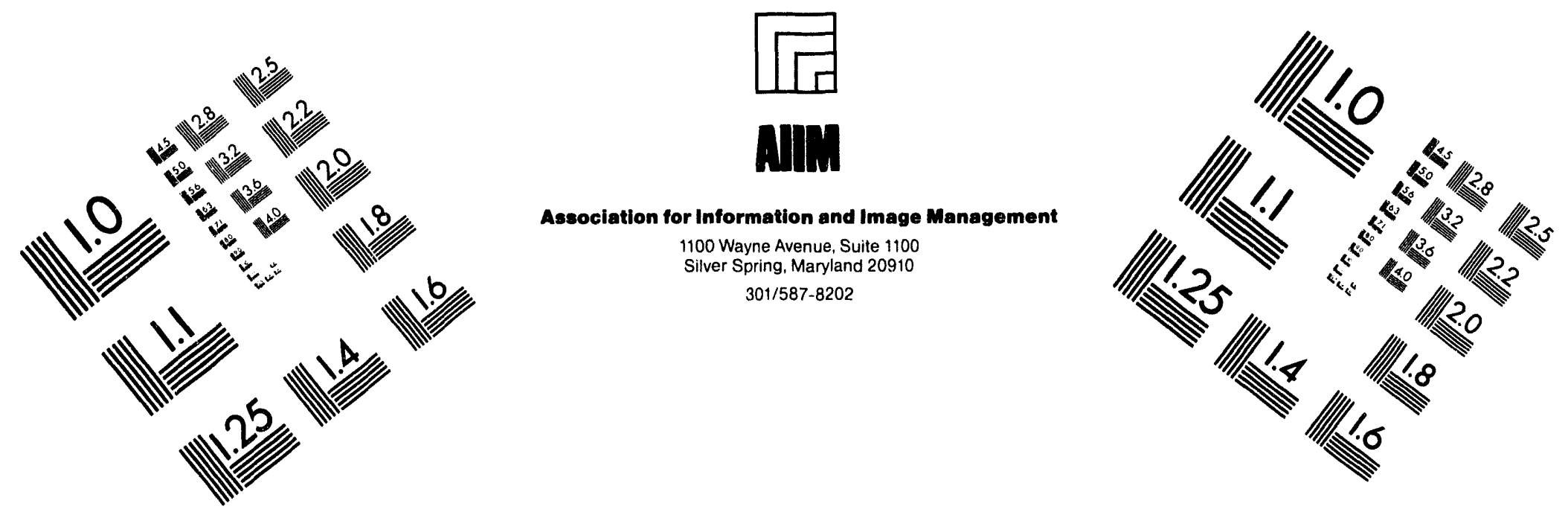

Centimeter

$\begin{array}{llllllllllllllll}1 & 2 & 3 & 4 & 5 & 6 & 7 & 8 & 9 & 10 & 11 & 12 & 13 & 14 & 15 & \mathrm{~mm}\end{array}$

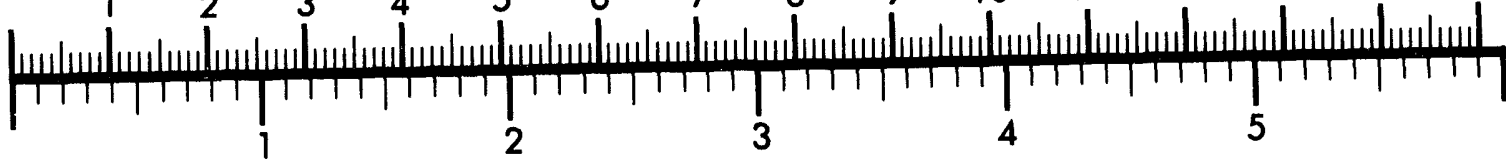
Inches

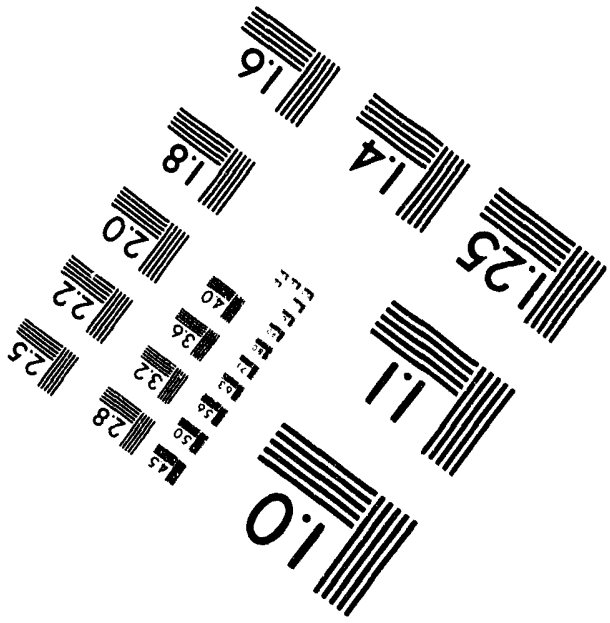

MANUFACTURED TO AIIM STANDARDS BY APPLIED IMAGE, INC.

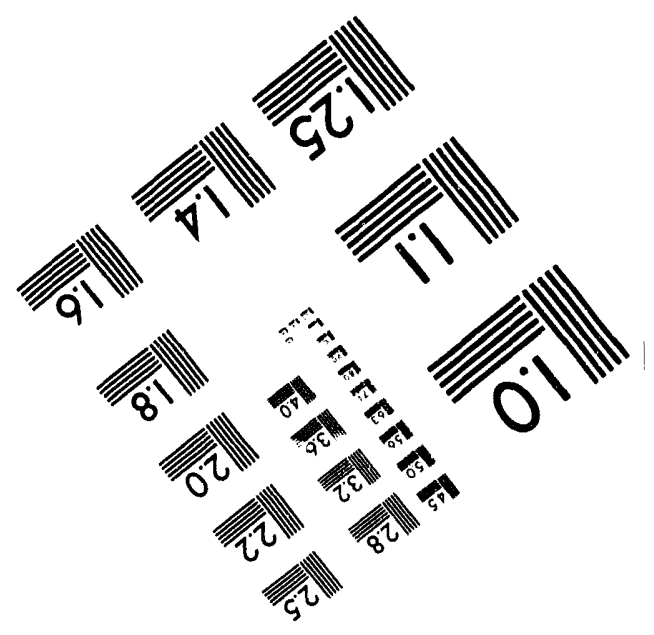





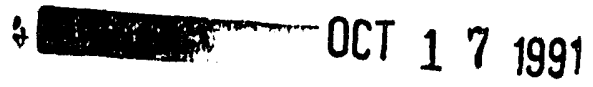

\section{STRUCTURAL BASIS OF SPECTRIN ELASTICITY}

Betty W. Shen," Steven B. Goldin," Fred J. Stevens, and Ursula Luthi

From: Biological and Medical Research Division, Argonne National Laboratory, Argonne, Illinois and 'Department of Biological Chemistry, University of Illinois at Chicago, Chicago Illinois.

Address: Biological and Medical Research Division

Argonne National Laboratory

9700 South Cass Ave, Argonne, Illinois 60439

* To whom correspondence should be addressed

The submitted manuscript has been authored by a contracior of the U.S. Government

under contract No. W.31.109ENG.38.

Accordingly, the U.S. Government retains a

nonexclusive, royalty-free license to publish

or reproduce the published form of this

U. S. Government purposes.

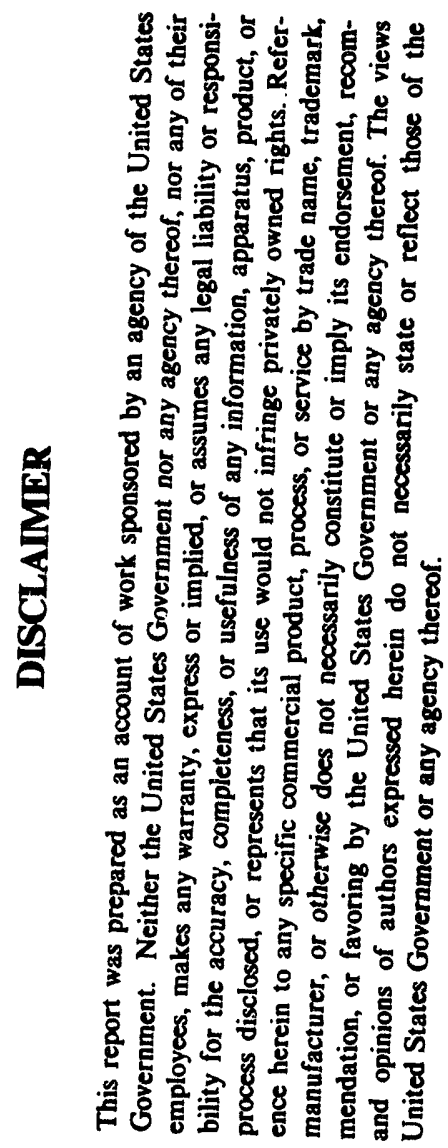

1 


\section{ABSTRACT}

A new model of human erythrocyte $\alpha$-spectrin is proposed. The secondary structure of human erythrocyte $\alpha$-spectrin and its folding into a condensed structure that can convert reversibly in situ, into an elongated configuration is predicted from its deduced protein sequence. Results from conformational and amphipathicity analyses suggest that $\alpha$-spectrin consists mainly of short amphipathic helices interconnected by flexible turns and/or coils. The distribution of charges and amphipathicity of the helices can facilitate their folding into stable domains of 4 and 3 helices surrounding a hydrophobic core. The association between adjacent four- and three-helix domains further organize them into recurring seven-helix motifs that might constitute the basic structural units of the extended $\alpha$-spectrin. The elongated spectrin molecule packs, in a sinusoidal fashion, through interactions between neighboring motifs into a compact structure. We suggest that the reversible extension and contraction of this sigmoidally packed structure is the molecular basis of the mechanism by which spectrin contributes to the elasticity of the red cell membrane. 


\section{INTRODUCTION}

Spectrin is the main component of a skeletal protein network that lines the cytoplasmic surface of the human erythrocyte membrane $(1-4)$. This network confers mechanical stability and elasticity on the human erythrocyte (5-8). The ability of spectrin to contribute to the mechanical stability of the skeleton arises from its dimer-dimer interactions, which lead to the formation of tetramers, the functional units in situ $(9,10)$, and from its interactions with actin, protein 4.1 , and ankyrin, which serve to organize and anchor the network to the membrane bilayer (11-15). How spectrin contributes to the elasticity of the skeleton is unknown. Several hypotheses and structural models have been proposed that relate the elasticity of the skeletal network to the physical and chemical properties of spectrin (16-19), but, little attention has been focused on its structure-function relationships.

Spectrin is a heterodimer, consisting of $\alpha$ and $\beta$ subunits associated in an antiparallel manner $(9,10,20)$. Both $\alpha$ - and $\beta$-spectrin are high-molecular-weight proteins (286,000 and 246,000 Da, respectively) consisting of homologous repeating sequences of approximately 106 amino acids $(21,22)$. Electron microscopy $(E M)$ of platinum shadowed specimens showed that purified spectrin dimers are long thin filaments approximately $1000 \AA$ in length (9). More recently, we have demonstrated by EM of negatively stained preparations showed spectrin can exist both as condensed and extended configurations in the intact skeleton (23). We suggested that the transition between these configurations could be the basis of membrane elasticity. 
In this study, we have examined the configuration of spectrin released under different experimental conditions and conclude that structural polymorphism is an intrinsic property of spectrin. This new structural information and the CDNA sequence reported recently (24) prompted us to initiate a new analysis of the secondary structure of human $\alpha$-spectrin. A new model emerged from our analysis which conforms to the known biochemical properties of spectrin, including the location of protease cleavage sites in normal spectrin (25) and in spectrin isolated from patients with hereditary anemia $(26,27)$. This model differs in many respects from the "three-helix bundle" model proposed by Speicher et al. $(22,25)$. Since erythrocyte spectrin is the most extensively characterized member of the spectrin gene family (2830), this model of an elastic biopolymer may have general bearing on our understanding of the structure and function of the spectrin family.

\section{Electron microscopy study}

The structure of spectrin released by different methods under various conditions was examined EM of negatively stained specimens over fenestrated grids. The EM specimens were prepared either immediately upon separation from the membrane bilayer or after prolonged dialysis against buffers containing dithiothreitol (DTT). Our results show that freshly released spectrin, either as free dimers and tetramers or as ensembles released together with protein 4.1 and actin, invariably assumed a condensed configuration regardless of the method and conditions under which it was released (Figure 1 left panels). However, manipulation of spectrin or spectrin- 
containing conglomerates, such as dialysis against DTT-containing buffer, column chromatography, or the addition of ammonium bicarbonate, all led to unfolding of the spectrin molecules to various extents (see Figure 1 right panels and the legend to Figure 1). Ammonium bicartonate is by far the most effective reagent for the unfolding of spectrin.

The above observations indicate that spectrin is in a condensed configuration in situ, which can convert to an elongated form upon its release from the bilayer. To understand the structural basis of this interconversion, we carried out conformational analysis of $\alpha$-spectrin based on secondary structures predicted from the protein sequence deduced from its CDNA sequence (24). The premises of our structure development lies on the assumptions that (i) folding of the secondary structures of $\alpha$-spectrin is governed by local hydrogen bonding and by hydrophobic as well as electrostatic interactions; (ii) folding is independent of the presence of $\beta$ spectrin; and (iii) the amphipathic character of the secondary structures plays an important role in the initiation and regulation of the folding of the polypeptide backbone (for reviews on the folding of proteins, see 31-34 and references cited therein).

\section{Structure prediction}

The prediction of secondary structure was based on the statistical method of Chou and Fasman (33) using conformational parameters derived from the crystallographic structures of 64 proteins (34). A sliding window of four residues was 
used for all potential and amphipathicity calculations. Threshold values were set as 6 residues for the assignment of helices and $\beta$-strands and 2 consecutive residues for $\beta$-turns. At locations where a segment displayed the potential for more than one conformation, the preferences of our selection were $\beta$-turn $>\alpha$-helix $>\beta$-strand, based in part on the higher reliability of the $\beta$-turn predictions by the Chou-Fasman algorithm, and also on the high helical content of the spectrin determined by circular dichroism spectroscopy. Furthermore, we ignored single residue gaps in an otherwise continuous peak if, and only if, the residue in question did not exhibit a potential for other conformations.

The amphipathicity of the helices and $\beta$-strands were calculated based on the algorithm described by Eisenberg et al. (35), with slight modifications. In our calculation, the entire sequence is viewed as a continuous helix or $\beta$-strand with residue number 1 as a fixed reference point for the direction of the hydrophobic moment of successive 4-residue segments along the primary sequence. The amphipathicity of the predicted helix and $\beta$-strand is obtained by multiplying hydrophobic mornents with the predicted Chou-Fasman potential and is plotted with a cutoff value of 0.5 alongside the conformational potential in a normalized scale.

The first step in our conformational analysis was to generate a consensus sequence for all 23 homologous repeats of $\alpha$-spectrin using the alignment suggested by Moon and McMahon (36), which takes into consideration homologies between 
erythrocyte and non-erythrocyte spectrin. Our consensus sequence is essentially identical to that reported by Sahr et al. (24). Overall, the hydrophobic residues are more highly conserved than the hydrophilic and charged residues. Of the 15 best conserved residues ( $>50 \%$ occupancy), 11 are hydrophobic residues having a large side chain (1 Phe, 2 lle, 6 Leu, and 2 Trp); only three are charged (Asp, His and Lys), and one (Gly) is a neutral residue. However, if we take into consideration residues of similar properties, the distribution of charged residues is as highly conserved as that of the hydrophobic ones (18 hydrophobic and 15 hydrophilic and charged residues altogether). This suggests that the hydropathy of the amino acids may play an important role in the folding of the molecule.

When placed on the surface of a helix net, most of the hydrophobic residues in the consensus sequence have one or more of their six neighboring positions (i.e., positions $i \pm 1, i \pm 3$, and $i \pm 4$ with respect to the $i$ th residue) occupied by residues of similar property. This suggests that conformations represented by the consensus sequence are likely to be amphipathic if they are $\alpha$-helical. If this is the case, most of the hydrophobic surface of the helices must be shielded from the aqueous environment by the packing of secondary structures, since spectrin is not a transmembrane protein. Moreover, its energy of stabilization must derive mainly from the interaction of secondary structures because it lacks prosthetic groups and disulfide linkages, factors that contribute to the folding and stabilization of other soluble globular proteins. 
Results from conformational analysis of the entire 2429 amino acid residues of $\alpha$-spectrin show that more than $75 \%$ have a helical potential greater than unity and that segments with high $\beta$-turn potential invariably coincide with breaks in helical potential (Figure 2, panels A and B). Most importantly, we found that most, if not all, of the potential helices are amphipathic judging from its amphipathicity profile (Figure $2 C$ ), while only 6 relatively small peaks (6-8 residues each) in the entire $\alpha$-spectrin sequence display significant amphipathic $\beta$-strand potential (data not shown). This prediction of unusually high helical content and low $\beta$-strand content is in agreement with earlier results of circular dichroism spectroscopy measurements of spectrin (37) and with the prediction by earlier reports (22). However, the number and length of the helices predicted by our algorithm differ from that predicted by earlier studies (22). Since the potential $\beta$-strands are few and well separated, we focused our attention on the packing of the amphipathic helices.

The composite helical potential profile of $\alpha$-spectrin (Figure 2A) shows that the length and position of the putative helices are highly variable. Most helices are relatively short (7-20 amino acids), with an average length of 13 residues. Large breaks in the helical potential profile tend to occur at the boundary of the repeats, as well as close to the midpoint of the repeats dividing them into segments of unequal length. Additional breaks in the helical potential or peaks in $\beta$-turn exist throughout the entire sequence of the repeats. Since the position and length of the helices are highly variable among the repeats and because the spectrin molecule has an 
unusually high helical content, we decided to analyze the folding of individual repeats instead of collectively averaging the conformations among repeats, which has been the basis for the development of previous models.

Helical wheel projections (38) showed that the seven potential helices which span slightly more than the length of repeat number one are indeed amphipathic; that is, hydrophobic and hydrophilic residues segregate to different sides of the helices (Figure $3 A$ ). Since a combination of high helical potential and amphipathicity has been shown to facilitate the folding of designed synthetic peptides and native-like proteins into stable domains of antiparallel helices (39-42), we propose that these short helices of $\alpha$-spectrin also fold into stable domains of antiparallel helices driven by their amphipathicity. In predicting the packing of these amphipathic helices, we take into consideration (i) the direction and spatial disposition of consecutive helices predetermined by the short length of the connecting segments (43); (ii) the maximum number of hydrophobic interactions; and (iii) the minimum number of repulsions between hydrophobic and hydrophilic residues and between residues of like charge.

Results from the above prediction suggest that the first seven helices of the $\alpha$ spectrin can organize into two stable domains of four and three helices, respectively (Figure 3B, C). These domains are stabilized by both hydrophobic and electrostatic interactions. Most hydrophobic residues are at the interiors of the clusters while most hydrophilic residues are at the surfaces. The charged residues in the interior of the 
helix-clusters are invariably in the vicinity of residues with opposite charge, and hence contribute to the stabilization of these domains through the formation of salt bridges in hydrophobic pocket. The small number of hydrophobic residues that remain exposed at the surface of the clusters are segregated to the sides of the clusters that permit inter domain interactions (Figure 3C).

Preliminary results from energy minimization and molecular dynamic analysis using Macromodel on Silicon Graphics (IRIS 3000) showed that clustering of helices I to $\mathrm{IV}$, based on orientations predicted by helical wheel projections, locked the 8 residue segment IV into a stable helix conformation and reduced the total energy of the system by approximately $350 \mathrm{kcal} / \mathrm{mol}$. Hydrophobic and electrostatic interactions account for all of the changes. Even though the numerical value of the calculated free energy change is based on the initial states and is likely to vary as the initial conformation of the helices changes, this result suggests that antiparallel association of these amphipathic helices is energetically favorable and that our prediction is in agreement with energy constraints.

When similar guidelines were applied to predict the folding of the $\mathrm{N}$-terminal $80-k D a$ tryptic domain of $\alpha$-spectrin (i.e., $\alpha$ I domain) (21), we were able to identify putative domains of four short amphipathic helices followed immediately by domains of thive helices. The distribution of the remaining hydrophobic residues and charged residues at the surfaces of the domains, once again, predicts the association between 
consecutive four- and three-helix clusters into recurring seven-helix motifs. We suggest that these motifs are the basic folding units of spectrin.

The predicted folding of the entire $\alpha$ I domain of spectrin and its packing into a condensed configuration is shown in Figure 4. In predicting the packing of the sevenhelix motifs into the condensed configuration, we seek maximum interaction between opposite charges and minimum repulsion between similar charges on the basis that most, if not all, of the hydrophobic residues are involved in either intra-motif interactions or in the association of the $\alpha$ and $\beta$ subunits. Our results suggest that the association of the second seven-helix motif with the first motif changes the direction of the helix procession, causing it to fold back on itself. While incorporation of the third motif does not change the general direction of the double row of helices, the incorporation of the next motif once again reverses the general direction of the molecule. The ribbon in Figure 4 shows that, even though the peptide backbone follows a 'zig-zag' course and the overall appearance of the molecule is a disordered two-dimensional array of short amphipathic helices, the molecule follows a sigmoidal path.

Judging frorn the overwhelming distribution of amphipathic $\alpha$-helices and the conservation of charged and hydrophobic amino acids over the entire sequence, it is conceivable that similar patterns exist throughout the $\alpha$-spectrin molecule. We suggest, based on both the conformational analysis and EM studies, that the entire 
spectrin molecule organizes into stable domains of 4 and 3 amphipathic helices interconnected by flexible turns or loops. The consecutive domains may associate with each other and form seven-helix motifs. This extended motifs-on-a-string configuration can fold in a sinusoidal fashion leading to the formation of a disordered two-dimensional array of short amphipathic helices. The domains are stabilized by strong hydrophobic and electrostatic interactions whereas the interactions between motifs are rather weak and mostly electrostatic.

The folding of the helices into stable domains (or motifs) shortened the length of the extended chain of helices by approximately three-fold so that an extended motifs-on-a-string configuration conforms to the length oi the spectrin dimers measured in the photomicrographs of the platinum-shadowed preparations (9) and expanded skeletons in negatively-stained preparations $(23,45)$. The folding of the motifs-on-a-string configuration in a sinusoidal pattern further reduces the distance between the ends of the spectrin molecule and leads to the formation of highly compact structure. The dimension and condensed nature of this new spectrin model corresponds well with the calculated distance between neighboring actin protofilaments (ca. $600 \AA$ per tetramer) and with the granular appearance of spectrin molecules in situ (45) as well as in skeletons freshly released from the bilayer (23).

Based on the assumption that spectrin is responsible for the solid elastic behavior of the red cell membrane, Waugh et al. (46) estimated, from the 
thermodependence of the red cell elastic modulus, an energy of ca. $0.6 \mathrm{kcal} / \mathrm{mol}$ for the extension of spectrin, which is, as indicated by the authors, much lower than the energies of hydrophobic, electrostatic, and metabolic effects. The above authors also showed that the shear elastic modulus of red cell membrane decreases with increasing temperature, suggesting that the deformed spectrin network is in a state of greater disorder and higher energy than the undeformed network (47). Judging from the electrostatic nature of the potential interactions involved in the folding of the condensed structure in our model, the energy required for extension and contraction of such a sinusoidal structure should not be excessive.

Since the spectrin tetramer is attached to the membrane bilayer at wellseparated sites along the entire length of the tetramer, it loses its freedom of rotation and is expected to retain energies generated due to extension of the molecule resulting from deformation of the membrane by shear stress. The energy which is stored as chemical potential is sufficient to facilitate the refolding of the elongated molecule once the external shear stress is removed. The dissipation of energy through inter motif rotation of molecule in solution may partially account for our lack of success in refolding of the extended spectrin molecule following its release from the bilayer (unpublished result). The fact that spectrin undergoes conformational changes once released from the bilayer may also account for the discrepancies between results observed by Stokke et al. on the thermodependence of the intrinsic viscosity of isolated spectrin dimers (48) and those reported by Waugh et al. on the 
thermoelasticity of red cells $(46,47)$.

\section{DISCUSSION}

A model consisting of repeating three-helix bundles was initially proposed for spectrin based on the repeating sequences of the $\alpha I$ domain (22) and the appearance and physical length of the spectrin filaments determined by EM studies (9). In this early model, it was proposed that the 106 amino acid repeats fold into three long helices of approximately 33 residues forming interconnected helical bundles with their long axis parallel to the length of the filamentous molecule and to the plane of the bilayer. This "three-helix bundle" model was later developed into a "rod-shaped" model for dystrophin (31). However, neither filaments nor rigid rods of $1000 \AA$ can be detected in the native skeleton without expansion of the network. In addition, this simplified model is inconsistent with properties of spectrin derived from protease digestion and mutation studies. Recently, there have been disagreements over the number of helices and $\beta$-strands in each 106 amino acid repeat used for the derivation of the original "three-helix bundle" model $(49,50)$, and a model consisting of three helices and two $\beta$-strands was proposed based on collective averaging of secondary structures predicted from a partial sequence of human $\alpha$-spectrin (49). However, there has been very little discussion on the validity of this new model in terms of tryptic digestion or properties of spectrin.

We have based our analysis on the availability of the complete protein 
sequence, the compact structure of spectrin in situ and the conformational rearrangement of spectrin upon its release from the bilayer, as well as on a set of more precise conformational parameters compiled from the structure of 64 proteins (34). No assumptions were invoked aside from a set of rules deduced from the folding of globular proteins. Several inferences can be drawn from the current structural prediction. (i) Spectrin consists of mainly short amphipathic helices connected by $\beta$-turns and nonperiodic coils (or loops). It is interesting that there are two segments (in repeats number 10 and 13) that display a potential helix/ $\beta$-strand/helix structure. The significance of these structures is not clear. (ii) The sequence homology of the 106-amino acid-repeats is only partially reflected in the putative secondary structure. Even though most of the hydrophobic residues having large side chains are situated in the interior of the domain and all but one of the most highly conserved Trp-lle pairs (at positions 45 and 46 of the 106 residue repeating unit) are in a helical environment, the boundaries of the domains and seven-helix motifs in our model do not correspond with those of the repeating units. (iii) The distribution of amino acids favors antiparallel association of consecutive helices, leading to the formation of stable domains of three or four helices surrounding a hydrophobic core. (iv) Domains that contain a fair number of exposed hydrophobic residues are in the range of inter domain interactions. (v) Both hydrophobic and electrostatic interactions contribute to short-range inter-domain interactions but only electrostatic interactions are involved in long-range interactions. 
It is important to point out that the boundary of our seven-helix motif runs across and shows little correlation with the boundaries of the 106 amino acid repeats. However, preliminary analysis shows that the sequence of domain I is highly homologous to the sequence of domain III (56\% similarity and $25 \%$ identity). It is possible, that the loosely conserved sequence homology among the repeating 106amino-acid segments of spectrin is designed to preserve the amphipathicity of the short helices which, in turn, contributes to the formation of stable domains of helices that constitute the functional units of spectrin elasticity.

The striking differences between the current model and the one previously proposed by Speicher et al. (22) are the length and arrangement of helical segments in the folding units. The helices in our model are substantially shorter than the ones predicted by the earlier model ( 13 versus 33 residues for the average length of the helices) and the direction of the helical axes are variable with respect to both the direction of the molecule and the plane of the lipid bilayer. It is conceivable that some of the shorter segments might not be energetically stable as an isolated helix, but, the energy of stabilization of the domains and motifs as a whole should favor the formation of those short amphipathic helices. Moreover, even though the helices are depicted through out our discussion as helical wheels projected down the helix axis, the actual direction of the helices could be significantly deviated from $90^{\circ}$ to the plane of the bilayer. Whether the procession of helices wraps around a central axis while propagating down the sigmoidal course, a prerequisite for a coil-coil interaction with 
the $\beta$ subunit as suggested by McGough and Josephs (51), cannot be addressed at this stage.

Our model predicts that most lysine and arginine residues are involved in both intra- and inter-domain salt bridge formation in a rather condensed structure and thus are inaccessible to tryptic digestion. This may explain the resistance of spectrin to trypsin digestion. Our model is also consistent with results from altered tryptic digestion patterns resulting from naturally occurring mutations in patients with hereditary anemia $(26,27)$. For instance, insertion of leucine between positions 153 and 154 in spectrin of patients with hereditary elliptocytosis (26) shitts the amphipathicity of helix VIII by placing two positive charges in the hydrophobic region of domain III, which may destabilize and perhaps even lead to the rearrangement of domain III. Since domain III is immediately adjacent to $\operatorname{Arg}^{137}$ at the beginning of helix VII, such destabilization may render this residue susceptible to tryptic digestion. Furthermore, substitutions of Pro for Leu ${ }^{260}$ or $\operatorname{Ser}^{261}(27)$ at a tight $\beta$-turn between two antiparallel helices would disrupt hydrogen bonding in the turn and render Lys ${ }^{257}$ at the end of helix XII susceptible to tryptic digestion. Finally, substitution of GIn at position 471 by Pro (27) would disrupt helix XXIII and render $\operatorname{Arg}^{468}$ at the beginning of the helix susceptible to trypsin. It is interesting that all trypsin sensitive-sites (including the lysine residues at positions 6,16 , and 601 and $\mathrm{Arg}^{45}$ of $\alpha$ I domain) and the aforementioned tryptic sites in abnormal spectrins are all located at the bottom of the model depicted in Figure 4. This suggests that the association between the $\alpha$ - and $\beta$ - 
subunits occurs through interaction of the $\beta$-subunit with the upper half of the molecule.

Recently, an elegant study by W. T. Tse and coworkers (52) showed that a point mutation close to the $\mathrm{C}$-terminal of the $\beta$-spectrin gene resulted in a change in the tryptic susceptibility of $\mathrm{Lys}^{48}$ in the $\alpha$ d domain. It is possible that the single amino acid substitution (Ala ${ }^{\beta-2054} \rightarrow$ Pro) may not only disrupt the folding of the last four-helix domain in the $\beta$-spectrin (unpublished results) but also alter the three-dimensional structure of the first seven-helix motif in the $\alpha$-spectrin resulting in the exposure of Lys $^{\alpha-4 \theta}$ that would otherwise be buried in the crevice between the first two domains in $\alpha$-spectrin.

Even though our model is self-consistent and compatible with existing data on the physical and chemical properties of the spectrin, questions remain as to the accuracy of our structural predictions. A small number of Arg and Lys residues in the $\alpha$ I domain that are predicted to be in flexible regions at the bottom of the molecule should be, according to our argument, susceptible to tryptic digestion but, in fact, remain intact after mild tryptic digestion. It is easy to argue that uncertainty in the prediction of the position of helices could be as much as three residues, which can explain the trypsin resistance of Lys or Arg residues in some of the shorter turns. However, most of the trypsin-resistant cleavage sites are in relatively long flexible loops. One plausible explanation is that minor structural components such as $C_{10}$ or $\Pi$ 
helices might exist in those long flexible regiens and alter the accessibility of the susceptible residues; or, we might have over- or under-predicted the number of helices resulting in an inversion of portions of the model. However, if our assumption is correct that charge distribution and amphipathicity of the helices governs the folding of spectrin, the general principle of the folding pattern and the dynamic property of the predicted model should prevail even if the direction of some of the helices are reversed and the residues in contact are different from the residues presented in Figure 4. Finally, association with the $\beta$-subunit may impose additional fine tuning on the structure of the $\alpha$-spectrin which can not be foreseen without detailed analysis of the interaction between domains and without better understanding of the nature and location of interactions.

Spectrin is the most extensively characterized member of a gene family that codes for high-molecular-weight proteins in a variety of cells. This family of proteins plays important roles in the organization, stability, regulation, and assembly of plasma membranes (for review, see reference 15). Increasing evidence suggests that spectrin adapts its diversified yet interrelated functions through specific interactions with both cytosolic and integral membrane proteins at different sites throughout its sequence. It is important to understand the structure of spectrin in order to delineate the mechanism by which this multivalent macromolecule exerts its function through intramolecular as well as intermolecular communications. Unfortunately, despite the wealth of information on the biochemical and biological properties of the molecule, 
structural information of spectrin remains scarce, mainly because of its large molecular mass and high flexibility. As a result, structural interpretation of biological functions must rely mainly on molecular models derived from the general principles of protein folding. We have presented here a testable model which is consistent with most, if not all, of the existing information on spectrin molecules and provides a structural basis for the elastic properties of the red cell membrane. Preliminary results using synthetic peptides confirm the amphipathic nature of the short helices. However, definitive proof of the model relies on clever design of peptide syntheses using the approach of Kaiser (39), Degrado(40), and Kaumaya (41), or the expression of selected regions of spectrin taking advantage of current advances in biomolecular technologies. Both approaches are feasible with the current stage of biotechnologies and are the major focus of many laboratories.

ACKNOWLEDGMENT: Work supported in part by grant HL 33254 from the National Institutes of Health (BWS) and in part by the U. S. Department of Energy, Office of Health and Environmental Research, under contract W-31-109-ENG-38. Electron microscopy of the released spectrin and spectrin-containing conglomerates were started initially as a collaboration with T. L. Steck of the University of Chicago. The authors thank Dr. Steck for discussion and critical reading of the manuscript and Dr. M. Kahn (University of Illinois at Chicago) for access to the IRIS 3000 Silicon Graphics. 


\section{REFERENCES}

1. G. L. Nicolson, V. T. Marchesi, and S. J. Singer, J. Cell Biol. 51, 265 (1971).

2. J. Yu, D. A. Fischman, and T. L Steck, J. Supramol. Struct. 1, 233 (1973).

3. J. Hainfeld, and T. L. Steck, J. Supramal. Struct. 6, 301 (1977).

4. M. P. Sheetz, and D. Sawyer, J. Supramol. Struct. 8, 399 (1978).

5. R. M. Johnson, G. Taylor, and D. B. Meyer, J. Cell Biol. 86, 371 (1980).

6. Y. Lange, R. A. Hadesman, and T. L Steck, J. Cell Biol. 92, 714 (1982).

7. M. Narla, J. A. Chasis, and S. B. Shohet, Semin. Hematol. 20, 225 (1981).

8. M. P. Sheetz, Semin. Hematol. 20, 175 (1983).

9. D. M. Shotton, B. E. Burke, and D. Branton, J. Mol. Biol. 131, 303 (1979).

10. S. C. Liu, and J. Palek, Nature (Lond.). 285, 586 (1980).

11. C. Cohen, Semin. Hematol. 20, 141 (1983).

12. V. T. Marchesi, Annu. Rev. Cell Biol. 1, 531 (1985).

13. D. Branton, C. Cohen, and J. Tayler, Cell 24, 24 (1981).

14. B. W. Shen, 'Red Blood Cell Membranes: Structure, Function, and Clinical Implications'. P. Aggre and J. C. Parker Eds. (Marcel Dekker, New York, 1989), pp. 261-297.

15. V. Bennett, Physiol. Rev. 70, 1029 (1990).

16. S. C. Liu, P. Windisch, S. Kim, and J. Palek, Cell. 37, 587 (1984).

17. J. M. Morrow, and V. T. Marchesi, J. Cell Biol. 88, 463 (1981).

18. J. S. Morrow, et al., Proc. Natl. Acad. Sci. USA 77, 6592, (1981).

19. A. Elgsaeter, B. T. Stokke, et al., Science 234, 1217 (1986). 
20. H. Yoshino, and V. T. Marchesi, J. Biol. Chem. 259, 4496 (1984).

21. D. W. Speicher, et al., J. Biol. Chem. 258, 14931 (1983).

22. D. W. Speicher, and V. T. Machesi, Nature (Lond) 311, 177 (1984).

23. B. W. Shen, R. Josephs., and T. L Steck, J. Cell Biol. 102, 997, (1986).

24. K. E. Sahr, et al., J. Biol. Chem. 65, 4434 (1990).

25. D. W. Speicher, G. Davis, and V. T. Marchesi, J. Biol. Chem 258, 14938 (1983).

26. S. L. Marchesi, et al., J. Clin. Invest. 80, 191 (1987).

27. M.-C. Lecomte, et al., Blood, 74, 1126 (1989).

28. M. Pearl, et al., J. Cell Biol. 98, 66 (1984).

29. M. D. Baron, M. D. Davison, P. Jones, and D.R. Critchley, J. Biol. Chem. 262, 17623 (1987).

30. M. Koenig, A. P. Monaco, and L. M. Kunkel, Cell 53, 219 (1988).

31. C. Chothia, M. Levitt, and D. Richardson, Proc. Natl. Acad. Sci. USA 74, 4130 (1977).

32. C. Chothia, M. Levitt, and D. Richardson, J. Mol. Biol. 145, 215 (1981).

33. P. Y. Chou, and G. D. Fasman, Biochemistry 13, 211 (1974).

34. G. D. Fasman, 'Prediction of Protein Structure and the Principles of Protein Conformation', D. G. Fasman ed. (Plenum Press, New York 1989), pp. 193-301.

35. D. Eisenberg, R. M. Weiss, and T. C. Terwilliger, Nature (lond), 299, 371 (1982).

36. R. T. Moon, and A. P. McMahon, J. Biol. Chem. 265, 4427 (1990).

37. G. B. Ralston, J. Supramolec. Struct. 8, 361 (1978). 
38. M: Schiffer, M., and A. B. Edmunson, Biophys. J. 7, 121 (1967).

39. E. T. Kaiser, and F. J. Kezdy, Science 223, 249 (1984).

40. W. F. DeGrado, Z. R. Wasserman, and J. D. Lear, Science 243, 622 (1989).

41. P. T. Kaumaya, et al., Biochemistry 29, 13 (1990).

42. M. H. Hecht, J. S. Richardson, et al., Science 249, 884 (1990).

43. J. S. Richardson, and D. C. Richardson, 'Prediction of Protein Structure and Principles of Protein Conformation', G. D. Fasman ed. (Plenum press, New York, 1989), Chapter 1, pp 1-98.

44. M. V. Nermut, Eur. J. Cell Biol. 25, 265 (1981).

45. T. J. Byers and D. Branton, Proc. Natl. Acad. Sci. USA 82, 6151 (1985).

46. R. Waugh, and E. A. Evans, Biophys. J. 26, 115 (1979).

47. D. A. Berk, R. M. Hochmuth and R. E. Waugh, 'R. d Blood Cell Membranes: Structure, Function, and Clinial Implications', Agre and Parker ed. (Marcel Dekker, 1989), Chapter 15, pp.423-453.

48. B. T. Stokke, A. Mikkelsen and A. Elgsaeter, Biochim. Biophys. Acta $816,102(1985)$.

49. Y. Xu, M. Prabhakaran, M. E. Johnson, and L. W.-M. Fung, J. Biomol. Struct. \& Dynam. 8, 55 (1990).

50. M. D. Davison, M. D. Baron, J. C. Wooton, and D. R. Critcchley, Int. J. Biol. Macromol. 11, 81 (1989).

51. A. McGough, and R. Josephs, Proc. Natl. Acad. Sci. USA. 87, 5208 (1990).

52. W. T. Tse, et al., J. Clin. Invest 86, 909 (1990). 
FIGURE 1: Electron micrographs of spectrin and spectrin containing conglomerates isolated under different conditions

Ghosts were prepared by hypotonic lysis of washed red cells as previously described (hypotonic ghosts), or by lysing washed red cells with $2 \mathrm{mM}$ Saponin in phosphate buffered saline, pH7 (PBS7 ghosts). Left panels: Samples applied to EM grids immediately after separation, Right panels: specimens applied to grids after extensive dialysis against $2 \mathrm{mM} \mathrm{NaPi} \mathrm{(pH} \mathrm{7.5)} \mathrm{containing} 0.5 \mathrm{mM} \mathrm{DTT}$. Bar $50 \mathrm{~nm}$.

Top: Spectrin containing conglomerates released from PBS7 ghosts by incubation with $2 \mathrm{mM}$ P-chloromercuriphenzylsulfonate (PCMBS) in PBS7 at $0^{\circ} \mathrm{C}$.

Middle: Spectrin dimers and tetramers released from PBS ghosts by high mechanical pressure (5000 psi) in a French press at $0^{\circ} \mathrm{C}$.

Bottom: Spectrin tetramers released from hypotonic ghosts.

FIGURE 2: Composite potential profiles for $\alpha$-spectrin homologous repeats The helix (Upper), and $\beta$-turn (Middle) potential profile together with the helical amphipathicity profile (Bottom) of human $\alpha$-spectrin are presented as 11 double repeats starting from residue number 14 . Repeat \#23 is not included in the alignment. The beginning of each double repeat is extended 9 residues toward the $\mathrm{N}$-terminal and, thus, is overlapped with the C-terminal of the preceeding double repeat. Each row in the panel represents a potential profile for a double repeat with cutoff values of 1.0 for $\alpha$-helix and $\beta$-turn and 0.5 for helical amphipathicity. 
FIGURE 3: Illustration of typical amphiphathic helices in human erythrocyte . $\alpha$-spectrin.

A. Helical wheel projections of the first four potential helices at the $\mathrm{N}$-terminal of human $\alpha$-spectrin. The arrows in helix number I (H1) represent the direction of the hydrophobic moment in the sliding 4-residue blocks along the primary sequence.

B. Predicted spatial arrangement of the four helices shown in $3 A$ and potential interactions between amino acids at the surface of different helices. C. Predicted packing of the first seven-helix motif at the $N$-terminus of human $\alpha$-spectrin. The color codes for positively and negatively charged residues are blue and red, respectively. While that for hydrophobic residues are yellow. A dot in the center of the helical wheel indicates that the C-terminal residue of the helix is pointed toward the viewer while a cross indicates that it is point away.

FIGURE 4: Predicted superstructural conformation of human erythrocyte $\alpha$-spectrin.

A. The packing of short helices into stable domains and the predicted interactions among domains in the $80 \mathrm{kDa}$ tryptic domain of spectrin ( $\alpha \mathrm{I}$ domain) is used as an example for the prediction of a superstructural conformation for human $\alpha$-spectrin. B. A simplified diagram of $\mathbf{4 A}$. The circles represent short amphipathic helices whereas the solid and dash lines represent the flexible segments connecting the helices (not in proportion to the number of residues in the segment). The ribbon depicts the general direction of the spectrin molecule. Colors same as figure 3. 

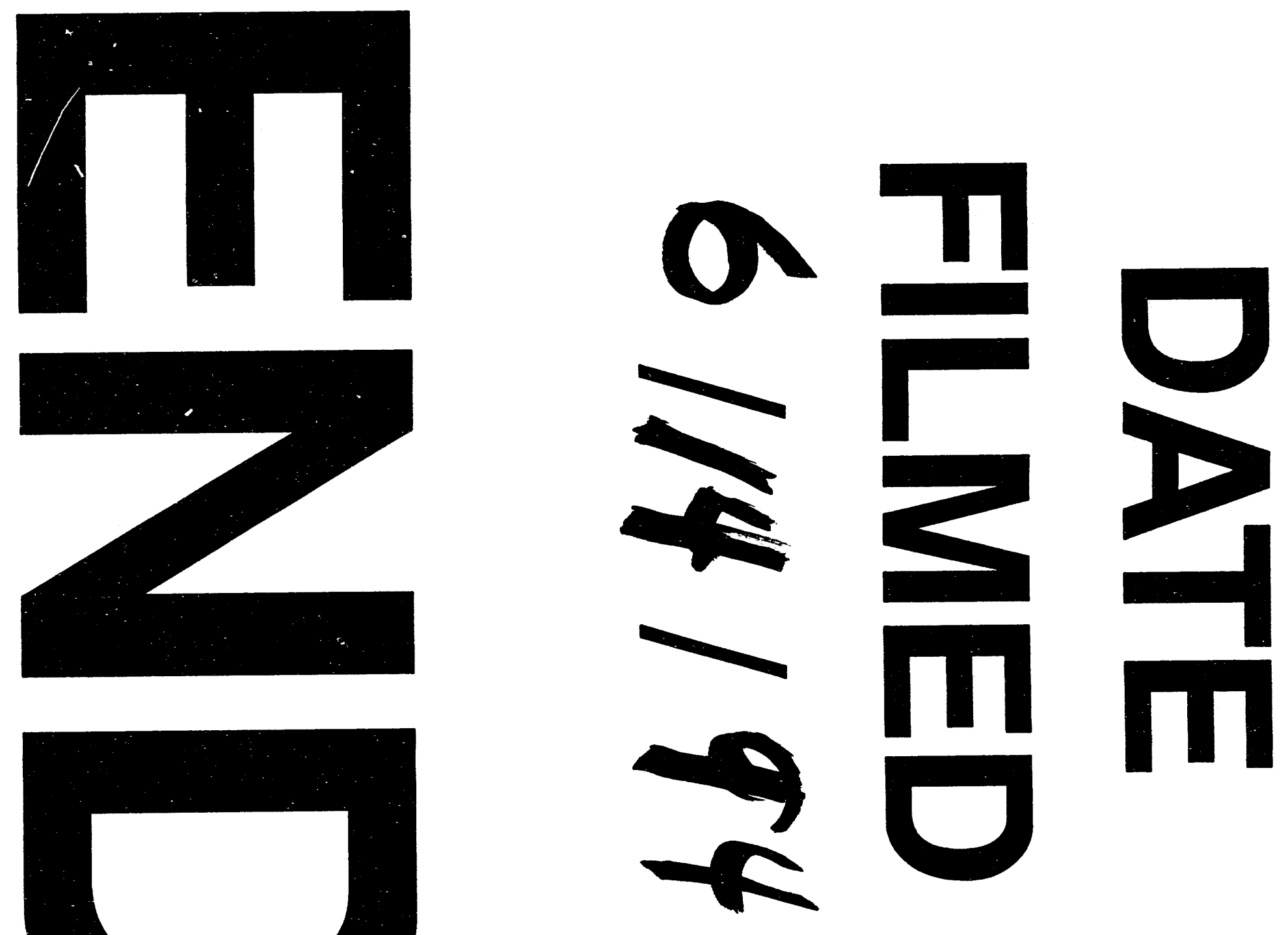
